\title{
Editorial
}

\section{Particle Swarms: The Second Decade}

\author{
Riccardo Poli, ${ }^{1}$ Jim Kennedy, ${ }^{2}$ Tim Blackwell, ${ }^{3}$ and Alex Freitas ${ }^{4}$ \\ ${ }^{1}$ Department of Computing and Electronic Systems, University of Essex, Colchester CO4 3SQ, UK \\ ${ }^{2}$ US Bureau of Labor Statistics, Washington, DC 20212 0001, USA \\ ${ }^{3}$ Department of Computing, Goldsmiths College, University of London, London SE14 6NW, UK \\ ${ }^{4}$ Computing Laboratory, The University of Kent, Canterbuy, Kent CT2 7NZ, UK
}

Correspondence should be addressed to Riccardo Poli, rpoli@essex.ac.uk

Received 7 April 2008; Accepted 7 April 2008

Copyright (c) 2008 Riccardo Poli et al. This is an open access article distributed under the Creative Commons Attribution License, which permits unrestricted use, distribution, and reproduction in any medium, provided the original work is properly cited.

\section{INTRODUCTION}

Particle swarm optimisation (PSO) was born just over ten years ago. The initial ideas on particle swarms of Kennedy and Eberhart were aimed at producing computational intelligence by exploiting simple analogues of social interaction, rather than purely individual cognitive abilities. The first simulations [1] were influenced by Heppner's and Grenander's work [2] and involved analogues of bird flocks searching for corn. These soon developed $[1,3,4]$ into a powerful optimisation method-the particle swarm optimiser.

In PSO, a number of simple entities-the particles-are placed in the search space of some problem or function, and each evaluates the objective function at its current location. Each particle then determines its movement through the search space by combining some aspect of the history of its own current and best (best-fitness) locations with those of one or more members of the swarm, with some random perturbations. The next iteration takes place after all particles have been moved. Eventually, the swarm as a whole, like a flock of birds collectively foraging for food, moves close to an optimum of the fitness function.

The particle swarm is more than just a collection of particles. A particle by itself has almost no power to solve any problem; progress occurs only when the particles interact. Problem solving is a population-wide phenomenon, emerging from the individual behaviours of the particles through their interactions. In any case, populations are organised according to some sort of communication structure or topology, often thought of as a social network. Each particle communicates with some other particles and is affected by the best point found by any member of its topological neighbourhood. The potential kinds of topologies of social networks are hugely varied, but in practice certain types, such as rings and fully connected networks, have been used more frequently.

\section{PARTICLE SWARM OPTIMISATION IS COMING OF AGE}

The particle swarm paradigm, that was only a few years ago a curiosity, has now attracted the interest of researchers around the globe. In fact, simple queries in publication databases, such as IEEE Xplore and Google Scholar, reveal that the number of publications in particle swarm optimisation has been exponentially growing for the last few years.

The aim of this special issue is to attract papers on particularly innovative algorithms, speculative ideas, new theoretical approaches, and novel applications that could act as seeds for PSO research in its second decade. Topics we solicited included the following: novel empirical and theoretical analyses of PSO population dynamics; innovative studies and algorithms for setting PSO parameters; new adaptive and parameterless PSO; analyses and new proposals of social network topologies; PSOs for combinatorial and hierarchical search spaces; novel PSOs for dynamic problems, noisy functions, and multimodal functions; advanced barebones/distribution-based PSOs; unconventional hybrids of PSO with other techniques; as well as novel applications in engineering, biomedicine, clustering, classification, entertainment, finance, image and signal processing, graphics, computational intelligence, and robotics. Amazingly, as we will show in Section 3, we managed to attract high-quality submissions in almost all these areas. 
As a first step in the direction of identifying new ideas for the second decade, we proposed and organised a workshop entitled "Particle Swarms: The Second Decade" at the Genetic and Evolutionary Computation Conference (GECCO) held in London in July 2007. Seven papers were selected by a peer-review process and presented at the workshop. The level of attendance was very good, the ideas presented were exciting, and the discussion that followed them was very lively.

Encouraged by this first success, we felt that we had to follow this on with a second, higher-impact initiative: this special issue of the Journal of Artificial Evolution and Applications. All of the presenters at the GECCO workshop were invited to extend their work and submit to this special issue, although the special issue was also open to new contributions. The special issue was a resounding success, attracting a total of 50 high-quality submissions. After a rigorous multistage review process, just under 20 papers were eventually accepted, and are now presented in this special issue. (Following the usual principles of blind review, papers coauthored by one of the guest editors were handled by another editor, so the former did not know which reviewers were assigned to his paper and had no influence on the editorial decision about the paper.)

\section{THE PAPERS IN THIS SPECIAL ISSUE}

We have chosen to divide the articles in this special issue into three main categories (although some spanned more than one): innovative theoretical/empirical analyses and theoretically sound design (Section 3.1), new exciting types of particle swarms (Section 3.2), and novel applications (Section 3.3). We will briefly introduce them in the next subsections.

\subsection{Theory, analysis, and principled design of particle swarms}

After a decade of relatively slow progress, the theory of PSO is now making significant and rapid progress, and this trend is likely to continue in the second decade. Indeed, four papers in this special issue deal with either theoretical explanations or theoretically driven design of PSOs.

The success or failure of stochastic optimisation algorithms depends on their ability to explore the search space associated with a problem effectively. The search is controlled by what is known as the sampling distribution of the optimiser. The article "Dynamics and stability of the sampling distribution of particle swarm optimisers via moment analysis" by R. Poli looks at the precise identification of the sampling distribution and its changes over time for standard forms of PSO. The article "Examination of particle tails" by T. Blackwell and D. Bratton focuses also on the characterisation of the way PSOs sample the search space, but, in this case, the analysis coarse-grains over the time variations of the distribution; the resulting distribution is shown to follow a power law.

One important source of innovation in PSO is the extension of the paradigm to the exploration of discrete search spaces. This is difficult because certain notions, such as the notion of velocity, are not easily extended to such spaces. So, until now, designing discrete PSOs has been essentially a black art. In this special issue, however, we are fortunate to have two articles- "Geometric particle swarm optimisation" by A. Moraglio et al. and "Forma analysis of particle swarm optimisation for permutation problems" by T. Gong and A. L. Tuson-which provide two different general approaches to derive theoretically sound PSOs for generic discrete search spaces.

\subsection{Novel particle swarms}

Broadly speaking, a particle swarm has two elements: particle dynamics and a mechanism for the sharing of information. The dynamics tells a particle how to move given the information that it has available; the result of this movement is subsequently communicated to other particles. These components, which mutually interact to deliver a swarm's searching capability, can be modified in various ways to derive new particle swarms.

Novel particle dynamics are represented here by a number of papers. S. Kok and J. A. Snyman in "A strongly interacting dynamic particle swarm optimization method" consider how particle dynamics can be affected by both position (as in standard PSO) and function value at that position, thereby introducing gradient information explicitly into the update rules. In a novel approach, J. L. FernándezMartínez and E. García-Gonzalo consider how PSOs can be derived from discretisations of continuous particle trajectories. Their paper "The generalized PSO: a new door to PSO evolution" presents theoretical stability analysis and experimental testing. A general trend in the work represented by these two papers is a growing interest in considering a swarm as an interacting system of "physical" particles, that is, as physical entities with continuous trajectories, momentum, energy dissipation, and force laws.

Two more papers consider alternative dynamics. In "Novel orthogonal momentum-type particle swarm optimization applied to solve large parameter optimization problems," J.-L. Liu and C.-C. Chang introduce an alternative velocity update rules with a "delta momentum" rule. They combine this rule with fractional factorial design for efficient optimisation. In "A simplified recombinant PSO," D. Bratton and T. Blackwell consider a series of simplifications to a biologically motivated dynamics based on genetic recombination. They demonstrate that velocity can be eliminated from the algorithm altogether, thereby making contact with distribution-based PSOs ("barebones" formulations).

The final two papers in this section consider how biological metaphors can induce changes to swarm algorithms at the population level. In "What else the evolution of PSO is telling us," L. Diosan and M. Oltean suggest changes to the order and frequency by which particles are updated. They use a genetic algorithm to explore various strategies. In "Particle swarm optimization for multimodal functions: a clustering approach," A. Passaro and A. Starita use k-means clustering to establish subswarm "niches." This approach involves dynamic information topologies 
that are linked to local spatial behaviour, features that are surely likely to be the subject of much second decade work.

\subsection{Applications}

Particle swarm optimisation has been enormously successful at solving problems of practical interest. This is reflected in this special issue by approximately half the articles reporting novel and exciting applications for PSO. We briefly review them below. Before we start, however, we would like to emphasise that several of the particle swarms proposed in the articles mentioned in this section are discrete PSOs, a characteristic shared by two theoretical approaches mentioned in Section 3.1. This indicates how prolific and important the area of discrete PSOs is becoming. We expect this area to grow significantly in the second decade.

The article "Optimizing the operation sequence of a multi-head surface mounting machine using a discrete particle swarm optimization algorithm" by Y.-M. Chen and C.-T. Lin, for example, describes how PSO can be used to optimise the process of picking and placing components onto printed circuit boards. R. Armellin and M. Lavagna's article entitled "Multidisciplinary optimization of aerocapture maneuvers" shows how PSO can design maneuvers that ensure that a spacecraft is captured by the gravitational attraction of a planet, with minimal expenditure of energy.

In "A hybrid PSO/ACO algorithm for discovering classification rules in data mining," N. Holden and A. A. Freitas extend a PSO algorithm with ideas borrowed from ant colony optimisation in order to cope with nominal (nonnumerical) variables, and apply the proposed algorithm to a data mining problem. In the article "An improved particle swarm optimizer for placement constraints," S.-T. Hsieh et al. show how PSO can solve floor-planning problems very effectively. With their paper entitled "Inverse parameter identification technique using PSO algorithm applied to geotechnical modeling," J. Meier et al. show how the PSO can provide valuable solutions in the difficult area of inverse-problem solving.

In "Particle swarm optimization for antenna designs in engineering electromagnetics," N. Jin and Y. Rahmat-Samii propose a PSO tailored to antenna design in engineering. The proposed PSO can cope with both real and binary variables, as well as multiobjective problems. In "Generating complete bifurcation diagrams using a dynamic environment particle swarm optimization algorithm," J. Barrera et al. apply PSO to the analysis of dynamical systems, which are represented as a set of equations specifying how variables change over time. In "A discrete particle swarm optimization algorithm for uncapacitated facility location problem," A. R. Guner and M. Sevkli propose a discrete PSO, as well as a hybrid version with local search, for solving a combinatorial optimization problem. In "Particle swarm for attribute selection in Bayesian classification: an application to protein function prediction," E. S. Correa et al. propose another type of discrete PSO algorithm to the problem of selecting attributes in data mining, and apply the algorithm to a bioinformatics problem.

\section{ACKNOWLEDGMENTS}

We would like to thank the Editor-in-Chief, Stefano Cagnoni, for his support in putting together this special issue. Cecilia Di Chio and Dan Bratton are also warmly thanked for their help with the local organisation of the GECCO workshop from which this special issue eventually emerged. The many reviewers who generously volunteered their time to help us with this special issue are also thanked. Finally, we would like to thank EPSRC (Extended Particle Swarms project, GR/T11234/01) for financial support.

Riccardo Poli Jim Kennedy

Tim Blackwell Alex Freitas

\section{REFERENCES}

[1] J. Kennedy and R. Eberhart, "Particle swarm optimization," in Proceedings of the IEEE International Conference on Neural Networks, vol. 4, pp. 1942-1948, IEEE Press, Perth, Australia, November-December 1995.

[2] H. Heppner and U. Grenander, "A stochastic non-linear model for coordinated bird flocks," in The Ubiquity of Chaos, S. Krasner, Ed., pp. 233-238, AAAS, Washington, DC, USA, 1990.

[3] R. Eberhart and J. Kennedy, "A new optimizer using particle swarm theory," in Proceedings of the 6th International Symposium on Micro Machine and Human Science (MHS '95), pp. 39-43, IEEE Press, Nagoya, Japan, October 1995.

[4] R. Eberhart, P. K. Simpson, and R. W. Dobbins, Computational Intelligence PC Tools, Academic Press Professional, Boston, Mass, USA, 1996. 